Original Research Paper

\title{
Development of a Short-Term Canine Full-Thickness Skin Organ Culture Method under Serum-Free Conditions
}

\author{
${ }^{1}$ Francesca Abramo, ${ }^{1}$ Andrea Pirone, ${ }^{1}$ Carla Lenzi, ${ }^{2}$ Maria Federica della Valle, \\ ${ }^{3}$ Silvia Vidali, ${ }^{1}$ Iacopo Vannozzi and ${ }^{1}$ Vincenzo Miragliotta \\ ${ }^{I}$ Department of Veterinary Sciences, University of Pisa, Viale delle Piagge 2, 56124, Pisa, Italy \\ ${ }^{2}$ Science Information and Documentation Centre (CeDIS), Innovet Italia SRL, Via Egadi 7, 20144 Milano, Italy \\ ${ }^{3}$ Department of Dermatology, University of Luebeck, Luebeck, Germany and \\ Department of Pediatrics, University Hospital Salzburg, Paracelsus Medical University, Salzburg, Austria
}

Article history

Received: 23-03-2016

Revised: 02-05-2016

Accepted: 10-06-2016

Corresponding Author: Vincenzo Miragliotta Department of Veterinary Sciences, University of Pisa, Viale delle Piagge 2, 56124, Pisa, Italy

Tel. $+39-050-2216865$

Fax: +39-2210655

Email: vincenzo.miragliotta@unipi.it

\begin{abstract}
Full-thickness canine skin organ culture models could provide an entirely new opportunity for studying wound healing, keratinization disorders and allergic skin diseases, all of which have high prevalence and severe impact on canine quality of life. Here we present a canine organ culture method for the short-term maintenance of full-thickness, adult, canine skin in serum-free medium and investigate the possibility to induce mast cell degranulation ex vivo. General morphological features were maintained up to day 7. Epidermal thickness started to decrease from day 4 of culture. No changes were observed in epidermal pigmentation. Keratinocyte proliferation started to significantly decrease at day 7 . Immunostaining for cytokeratin 10, cytokeratin 14 and loricrin was evident from day 1 to day 7 . Compound 48/80 induced mast cell degranulation. This was the first attempt to establish a dog skin organ culture and document good preservation of most cutaneous structures till day 7. This method may help in dissecting canine skin biology in physiological and pathological conditions and to study drug mechanism of action in a biologically relevant environment.
\end{abstract}

Keywords: Organ Culture, Skin, Dog, Histology

\section{Introduction}

Important advances in veterinary dermatology have been made over the past several years. Increased understanding of pathophysiologic mechanisms of allergic skin disease is one of the best examples (Marsella et al., 2011; 2012; Campora et al., 2012; Abramo et al., 2014). Yet, much remains to be done and future progress is likely to depend upon a better knowledge of skin biology and pathophysiological pathways. Although in vivo studies on the target species would obviously be among the most suitable methods, they can result in pain and discomfort, a critical factor given the growing ethical concerns on the use of animals for biomedical research. Indeed, the recent Directive 2010/63/EU specifically requires dissemination of best practice in animal research with particular emphasis on the 3 Rs (reduce, refine, replace). Thus, in vitro and ex vivo skin models could be a valuable alternative. Canine keratinocyte cultures have been developed
(Kimura et al., 2012; Shibata et al., 2010), but their single cell-type nature and lack of multidimensional growth are important limitations in investigating basic and advanced dermatology issues. Three-dimensional cell cultures (i.e., keratinocyte-fibroblast co-culture and organotypic culture) that might overcome these limitations have been generated (Yagihara et al., 2011; Serra et al., 2007). However, their fidelity to the in vivo situation is limited, mainly because the surrounding cellular environment differs from real skin, i.e., the matrix is simplified and the role of the nervous and immune response is excluded. Canine skin equivalents have been developed recently (Serra et al., 2007), but their use in skin biology and pathology is still lacking. Canine epidermis on porcine dermal support has also been reported and might become an interesting investigative tool in the future (Cerrato et al., 2012). Human skin organ culture models have been described for some decades, dating to the early studies of Beaven and Cox (1965) as well as Sarkany et al. (1965). Ultrastructural studies in 
healthy cultured skin samples were subsequently reported (Prose et al., 1967) along with pathogenetic investigations (Caron, 1968). Despite the prolific number of studies which followed these initial experiments, together with more refined and reliable protocols in other species, canine skin organ culture models were never attempted. Cultured skin explants from dogs until now have been limited to either establishing keratinocyte cultures (Wilkinson et al., 1987) or to extract T-cells (Jassies-van der Lee et al., 2014). A single study in veterinary medicine reports on the histological similarities between human and swine skin and the establishment of Gottingen minipig skin organ cultures (Dame et al., 2008). Clearly, there is a need to develop and validate experimental procedures for explanted canine skin tissues which maintain their architecture and physiology.

Canine skin organ culture methods may offer unique opportunities not only for overcoming concerns on the use of animals for biomedical research, but also to study pathophysiologic and pharmacologic skin responses in a biologically relevant 3 -dimensional environment which preserves the same cell-cell and cell-matrix contacts and communication present in the intact tissue. In fact, canine skin organ cultures represent a valuable tool for gaining new insights into the histological and biological features of dog skin and a better understanding of the etiopathological mechanism (s) underlying skin diseases. Moreover, it may allow one to study the canine skin response to test agents during naturally and experimentally-induced skin disorders and provide information on drug absorption. For example, fullthickness canine skin organ culture models could provide a new opportunity to study wound healing, keratinization disorders and allergic skin diseases, all of which have a high prevalence and severe impact on canine quality of life (Xu et al., 2012). Previous analysis of human skin organ cultures comparing different media showed reduced cell death under conditions that maintain the natural tissue environment (serum-free medium) (Lu et al., 2007). Our aim was to establish a canine organ culture method for the short-term maintenance of full-thickness, adult, canine skin in serum-free medium.

\section{Methods}

\section{Dog Skin Organ Culture}

Normal skin was obtained from three adult mixed breed female donor dogs subjected to surgical procedures unrelated to dermatological conditions (removal of neoplastic lesions/lymph node). Written informed consent was obtained from each dog owner. Skin was collected at the periphery of the surgical site in order to avoid atrophic changes due to the presence of tumor.
Briefly, before anesthesia with propofol $(4 \mathrm{mg} / \mathrm{kg}$, intravenous) and isoflurane $2 \%$ in oxygen, tricotomy was performed and the surgical site aseptically prepared. During the surgical procedure a skin strip was collected and promptly immersed in isolation medium: 99\% Williams' E Medium-L-glutamine-free (W4128, Sigma-Aldrich S.R.L. Milan, Italy) supplemented with $1 \%$ antibiotic/antimycotic solution (A5955, Sigma-Aldrich) containing 10000 IU penicillin, $10 \mathrm{mg}$ streptomycin and $25 \mu \mathrm{g}$ amphotericin B per $\mathrm{mL}$; the sample was kept on ice until establishment of the organ culture.

To perform submerged skin organ cultures, full thickness $4 \mathrm{~mm}$ skin biopsy punches were collected after mechanical removal of excess subcutaneous fat with a scalpel. Three skin biopsies from each dog were immersed in $10 \%$ buffered formalin solution $(\mathrm{pH}$ 7.4) and taken as day (D) 0 samples. Triplicate cultures were established from each dog (three biopsies per time-point); skin biopsies were carefully placed into 6-well plates containing Williams'E medium supplemented with $1 \% 10000 \quad$ IU $\mathrm{mL}^{-1}$ penicillin/10 $\mathrm{mg} \mathrm{mL}^{-1}$ streptomycin (P4333, SigmaAldrich) $0.1 \% \quad 10 \mu \mathrm{g} \mathrm{mL}^{-1}$ insulin (I9278, SigmaAldrich), $0.02 \% 10 \mathrm{ng} \mathrm{mL}^{-1}$ hydrocortisone (H0135, Sigma-Aldrich), and 1\% $200 \mathrm{mM}$ L-glutamine (G7513, Sigma-Aldrich) (Lu et al., 2007). Medium was replaced after $24 \mathrm{~h}$ and then at 2-day intervals. Skin samples were kept at $37^{\circ} \mathrm{C}$ in a humidified incubator with $5 \%$ $\mathrm{CO}_{2}$ for 7 days. Three biopsy samples were collected and fixed in $10 \%$ buffered formalin solution ( $\mathrm{pH}$ 7.4) for each selected time point (D1, 4 and 7) and routinely processed for paraffin embedding, thus allowing a time-course of the evolution of epidermal, dermal and adnexal conditions during culture.

\section{Morphological Evaluation}

Morphological features of submerged skin were evaluated on hematoxylin/eosin and Mallory stained 5 $\mu \mathrm{m}$ sections. The presence of culture-induced changes was qualitatively evaluated on epidermis, dermis and adnexal structures. Mast Cell (MC) presence and qualitative granulation state was evaluated on metachromatic toluidine blue stained sections.

\section{Morphometric Assessment of Epidermal Thickness and Pigmentation}

Epidermal thickness was measured on $20400 \mathrm{X}$ captured fields per time point, by manually tracing 16 segments at regular intervals per captured field with NIS-Elements $\mathrm{Br}$ Microscope Imaging Software (Nikon Instruments, Calenzano, Italy.). Segments were perpendicular to the basement membrane and extended from the germinative layer to the beginning of the stratum corneum. 
Skin pigmentation was quantitatively evaluated at the epidermal level in unstained sections; briefly, $600 \mathrm{X}$ fields were acquired (including the entire extent of epidermis) after removing the microscope condenser and a Region Of Interest (ROI) was manually defined; the ROI included the epidermis from the basement membrane to the beginning of the stratum corneum. Image segmentation was performed by manually defining a threshold (detecting the pigmented areas) with the NIS-Elements Br Microscope Imaging Software; the relative presence of melanin (melanin density) was calculated by dividing the measured area by the ROI area. The mean size of particles was also recorded.

\section{Keratinocyte Proliferation}

Ki67 immunostaining was performed to evaluate keratinocyte proliferation. Briefly, Superfrost-Plus mounted sections were deparaffinized and rehydrated. Epitope retrieval was carried out at $120^{\circ} \mathrm{C}$ in a pressure cooker for 3 min with a Tris/EDTA buffer $\mathrm{pH}$ 9.0. Quenching of endogenous peroxidases was carried out by incubation with $1 \% \mathrm{H}_{2} \mathrm{O}_{2}$ in $0.1 \mathrm{M}$ phosphate buffer $\mathrm{pH} 7.4$ (PBS) for $5 \mathrm{~min}$ at Room Temperature (RT). Non-specific binding was blocked by incubation of slides with $5 \%$ normal goat serum (in PBS 1 h, RT; Cat $\mathrm{N}^{\circ}$ s-1000; Vector Labs, Burlingame, CA, USA). Slides were then incubated with mouse monoclonal anti-Ki67 primary antibody (Cat $\mathrm{N}^{\circ}$ sc-101861; Santa Cruz Biotechnology, Inc. TX, USA.) diluted 1:200 in PBS overnight at $4{ }^{\circ} \mathrm{C}$. Subsequently slides were rinsed in PBS, $3 \times 10 \mathrm{~min}$, followed by incubation with a biotinylated goat antimouse immunoglobulin (Cat $\mathrm{N}^{\circ}$ BA-9200; Vector Labs), diluted 1:300 in PBS. Sections were again rinsed in PBS, for $3 \times 10 \mathrm{~min}$. Staining was visualized by incubating sections in diaminobenzidine (DAB; Cat $N^{\circ}$ sk-4105; Vector Labs) solution. Sections were then dehydrated, cleared and mounted with a permanent mounting medium. Five $250 \mathrm{X}$ fields were photographed per time point and Ki67-positive cells counted and expressed as percent of basal cells.

\section{Epidermal Differentiation Markers}

To assess epidermal differentiation cytokeratin 10 (suprabasal layers), cytokeratin 14 (all epidermal layers except the stratum corneum) and loricrin (granular layer) were immunolocalised (Xu et al., 2012). Indirect immunofluorescence was performed as follows: Superfrost-Plus mounted sections were deparaffinized and rehydrated; epitope retrieval was carried out as above. Non-specific binding was blocked by incubation of slides with PBS $+2 \%$ Bovine Serum Albumin (BSA; Cat. N SP-5050; Vector Labs) (45 min at RT). Slides were incubated overnight at $4^{\circ} \mathrm{C}$ with mouse monoclonal anti cytokeratin 10 (Cat
$\mathrm{N}^{\circ}$ ab9026; Abcam plc, Cambridge, CB4 0FL, UK), mouse monoclonal anti cytokeratin 14 (Cat $\mathrm{N}^{\circ}$ ab7800; Abcam plc) or rabbit polyclonal anti loricrin (Cat $\mathrm{N}^{\circ}$ ab24722; Abcam plc) diluted in PBS $+1 \%$ BSA. Subsequently, slides were rinsed in PBS, $3 \times 10$ min, followed by incubation with: fluorescein antimouse antibody (Cat $\mathrm{N}^{\circ}$ FI-2000; Vector Labs) for cytokeratin 14 and cytokeratin 10 and fluorescein anti-rabbit antibody (Cat $\mathrm{N}^{\circ}$ FI-1000; Vector Labs) for loricrin. Slides were then mounted with Vectashield mounting medium (Cat $\mathrm{N}^{\circ} \mathrm{H}-1500$; Vector Labs) with DAPI for nuclear counterstaining.

\section{Degranulation of Mast Cells by Compound 48/80}

Skin culture triplicates were also established to investigate the effect of compound 48/80 (C2313, Sigma-Aldrich) known to stimulate degranulation of skin MC (Wang et al., 2005; Lee and Kim, 2010). At D1 of culture, compound 48/80 was added to Petri dishes at concentrations of 0,5 and $10 \mathrm{mg} \mathrm{mL}{ }^{-1}$ for $20 \mathrm{~min}$. Samples were then collected and processed for paraffin embedding. One small $(1 \times 1 \mathrm{~mm})$ piece of tissue from each biopsy was collected and embedded in Epon Araldite resin. MC presence and qualitative granulating state was evaluated on metachromatic toluidine blue stained semi-thin sections $(1 \mu \mathrm{m})$.

\section{Statistical Analysis}

All data were analysed with GraphPad Prism (GraphPad Software, San Diego CA USA). One way ANOVA with Bonferroni's Multiple Comparison Test was used for statistical comparisons of the examined parameters per each time point.

\section{Results}

\section{Morphological Features}

Organ-cultured canine skin samples maintained an intact tissue architecture for the entire duration of the experiment (Fig. 1). The epidermis showed normal stratification with 2-3 nucleated layers and a cornified stratum corneum at D0, i.e., after $1 \mathrm{~h}$ incubation in isolation medium (Fig. 2a). While morphological qualitative differences were not observed at D1, at D4 the epidermis was only focally thinned to a 1-2 layer of keratinocytes (Fig. 2b) and showed occasional pyknotic keratinocyte nuclei both in the basal and spinous layers at D7 (Fig. 2c). Melanin was detected in the epidermis at each time point as small brownish to black dots in the keratinocytes of the basal and suprabasal layers and as aggregates in scattered melanocytes along the basal membrane (Fig. 2c, inset).

Dermis remained structurally intact and showed normal blue-stained collagen bundles with Mallory 
staining. The superficial, medium and periadnexal vascular plexuses were well represented throughout the duration of the experiment. Perivascular aspecific mononuclear cells were detected in all samples until D7, including MC. The latter were identified by their round to polygonal shape, a round central nucleus and presence of metachromatic cytoplasmic violet granules on staining with toluidine blue. Their presence was observed in both the superficial and deep dermis, as well as in close vicinity to hair follicles.
Hair follicles were well-preserved at D0 and displayed normal cycling morphology; scattered apoptotic figures of matrical cells as well as swelling and degeneration of the bulb wall cells were seen from D4. Sebaceous glands were well-preserved at D1 while from D4 some nuclei were irregularly shaped; cell borders became less visible at D7. Sweat gland epithelial cells were detached from their basal lamina at D4. Arrector pili muscles were well-preserved throughout the study period.
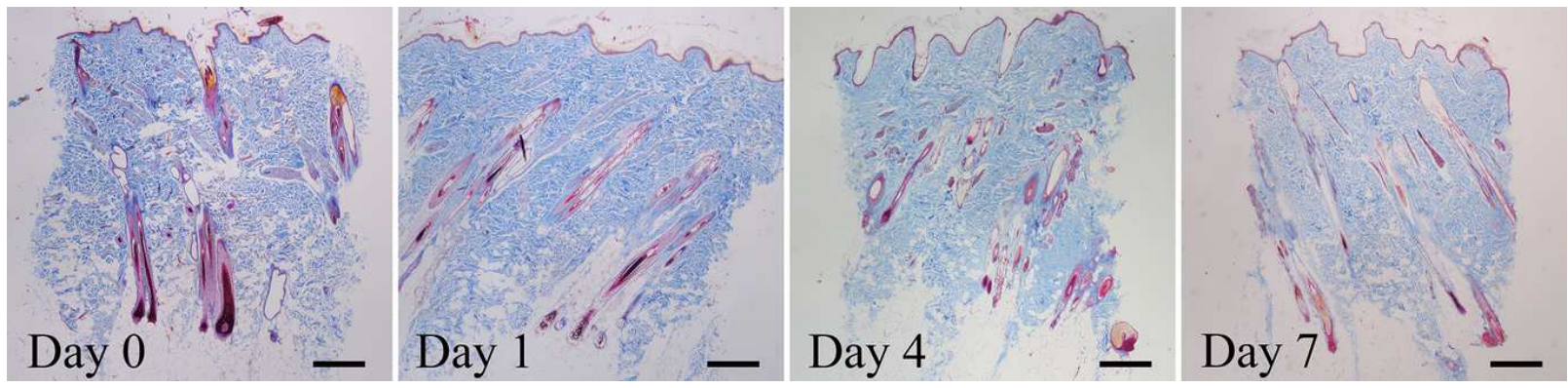

Fig. 1. Photomicrographs of cultured skin biopsies stained with Mallory's trichrome. The whole cultured biopsy was photographed to show the overall quality of epidermis, dermis and skin adnexa at different time-points of culture. Scale bar $=500 \mu \mathrm{m}$
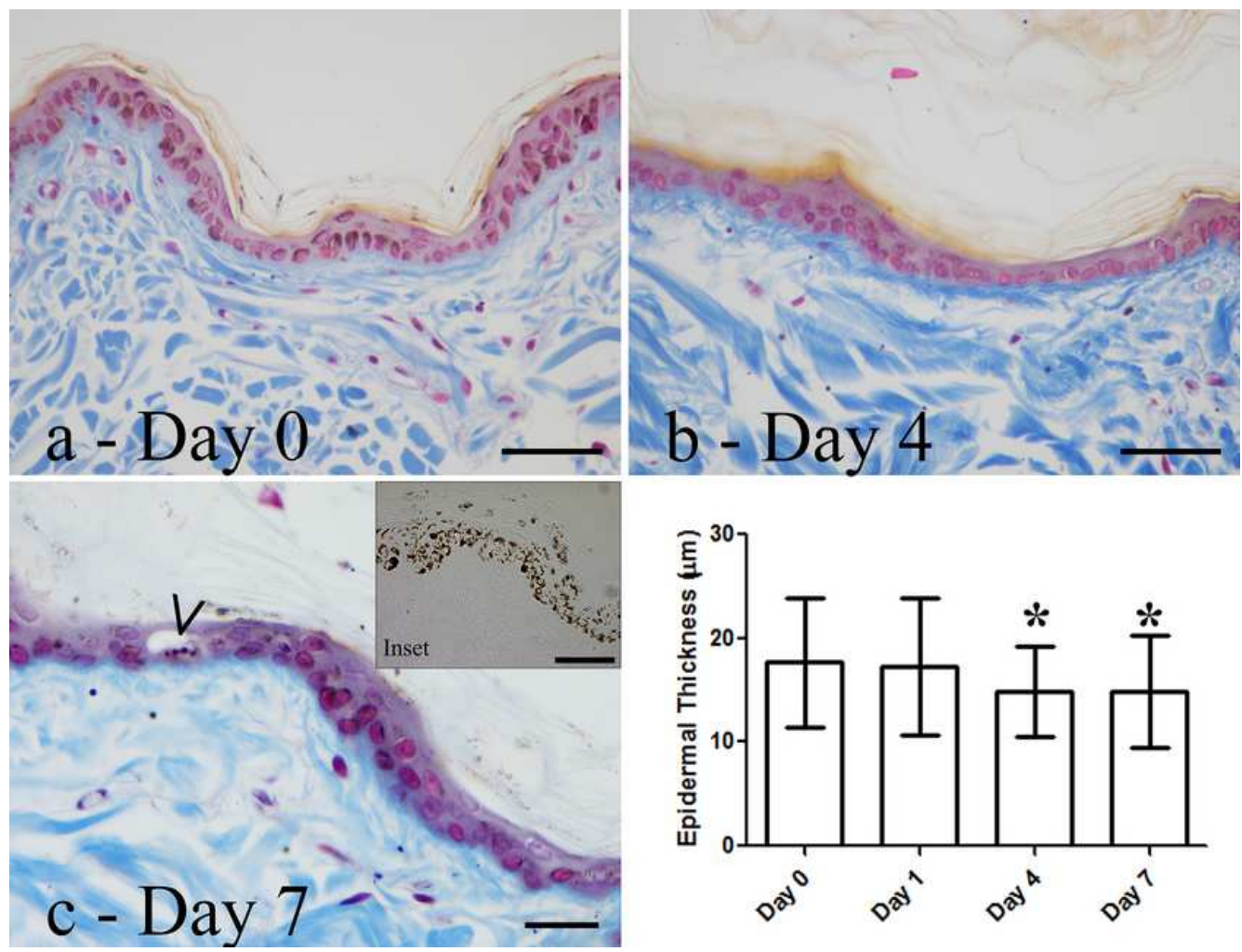

Fig. 2. Photomicrographs of cultured skin biopsies stained with Mallory's trichrome. The epidermis shows normal stratification with 2-3 nucleated layers and a cornified stratum corneum at day 0 (a), with only focally thinning to 1-2 layers of keratinocytes (b), pyknotic keratinocyte nuclei (arrowhead) were seen at D7 (c). Epidermal thickness (d) significantly decreased from day0/day1 to day4/day7 (asterisks; data presented as mean \pm SD). Inset shows a typical unstained section that was used for melanin content assessment. Scale bar: $50 \mu \mathrm{m}$ in a and b and inset; $25 \mu \mathrm{m}$ in c 


\section{Morphometric Assessment of Epidermal Thickness and Pigmentation}

Epidermal thickness $(\mu \mathrm{m})$ was $17.72 \pm 6.22$ at D0, $17.33 \pm 6.60$ at $\mathrm{D} 1,14.87 \pm 4.36$ at $\mathrm{D} 4$ and $14.85 \pm 5.42$ at D7; differences achieved statistical significance $(\mathrm{p}<0.001)$ between D0 and D4/D7; D0 did not differ from D1 and D4 from D7 (Fig. 2d).
Epidermal melanin density (pigmented area on epidermal area) showed no changes from D0 to D7 of culture. Melanin density was $15.47 \pm 6.06$ at D0, $15.64 \pm 4.6$ at $\mathrm{D} 1,17.14 \pm 7.40$ at $\mathrm{D} 4$ and $17.13 \pm 5.49$ at $\mathrm{D} 7$ with no statistically different changes. The mean size of measured objects $\left(\mu \mathrm{m}^{2}\right)$ was $2.65 \pm 1.23$ at $\mathrm{D} 0,2.42 \pm 0.94$ at $\mathrm{D} 1,2.79 \pm 1.28$ at $\mathrm{D} 4$ and $2.66 \pm 0.90$ at $\mathrm{D} 7$ with no statistically significant differences.
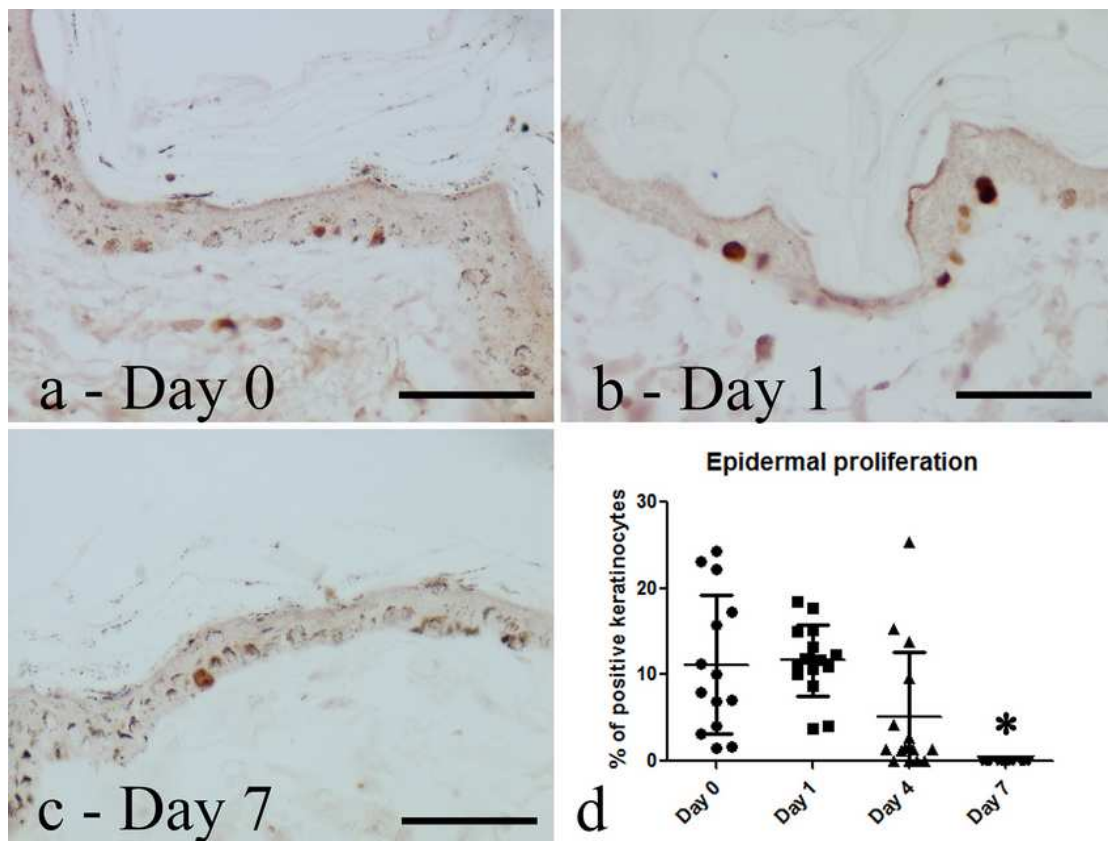

Epidermal proliferation

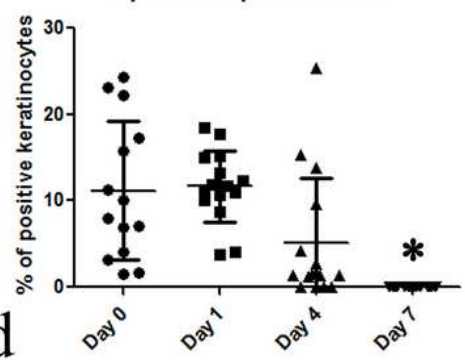

Fig. 3. Photomicrographs of Ki67 immunostained sections (a, b, c) and histogram representing Ki67 immunostaining quantitative analysis $(\mathrm{d})$. Asterisk $=$ statistically significant difference compared to day 0; data are means \pm SD. Scale bar $=50 \mu \mathrm{m}$

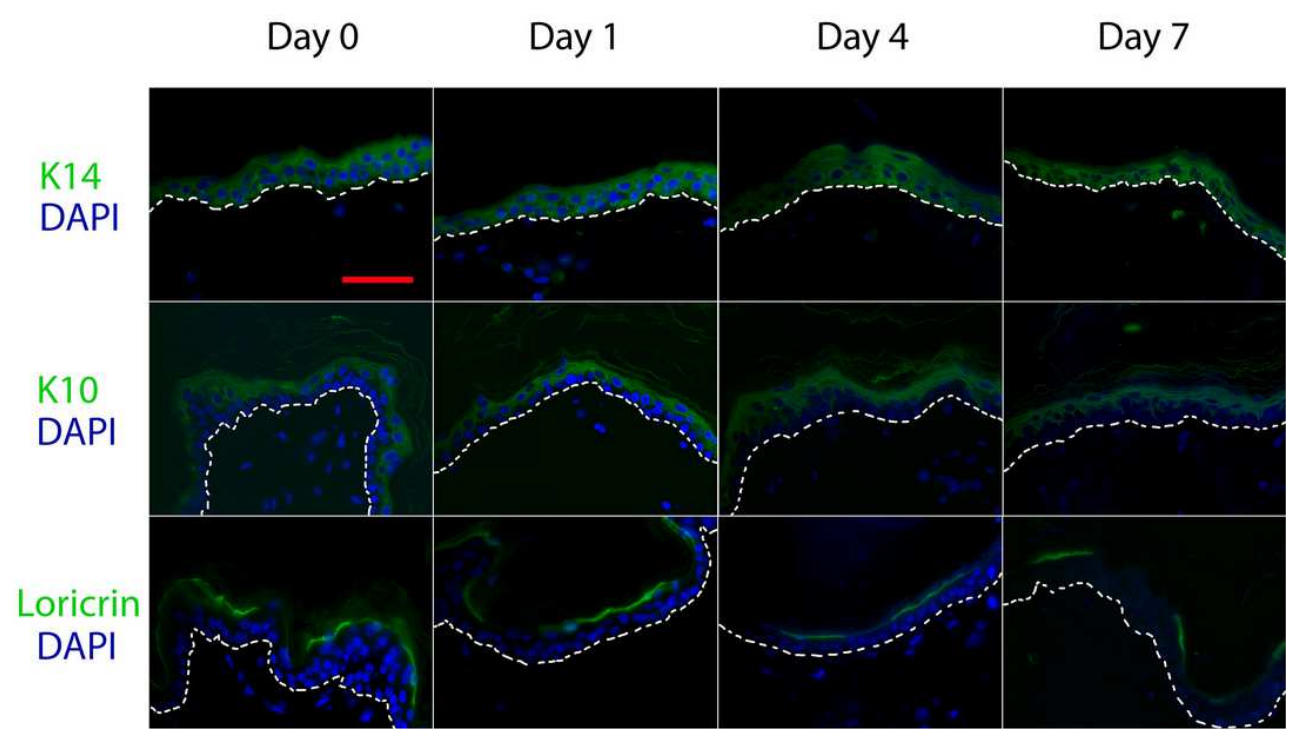

Fig. 4. Photomicrographs of cytokeratin 14 (top row) cytokeratin 10 (middle) and loricrin (bottom)-stained sections from day 0 (left) to day 7 (right). The day 0 pattern of staining was maintained for the study duration. Indirect immunofluorescence was counterstained with DAPI. Scale bar $=50 \mu \mathrm{m}$ throughout 

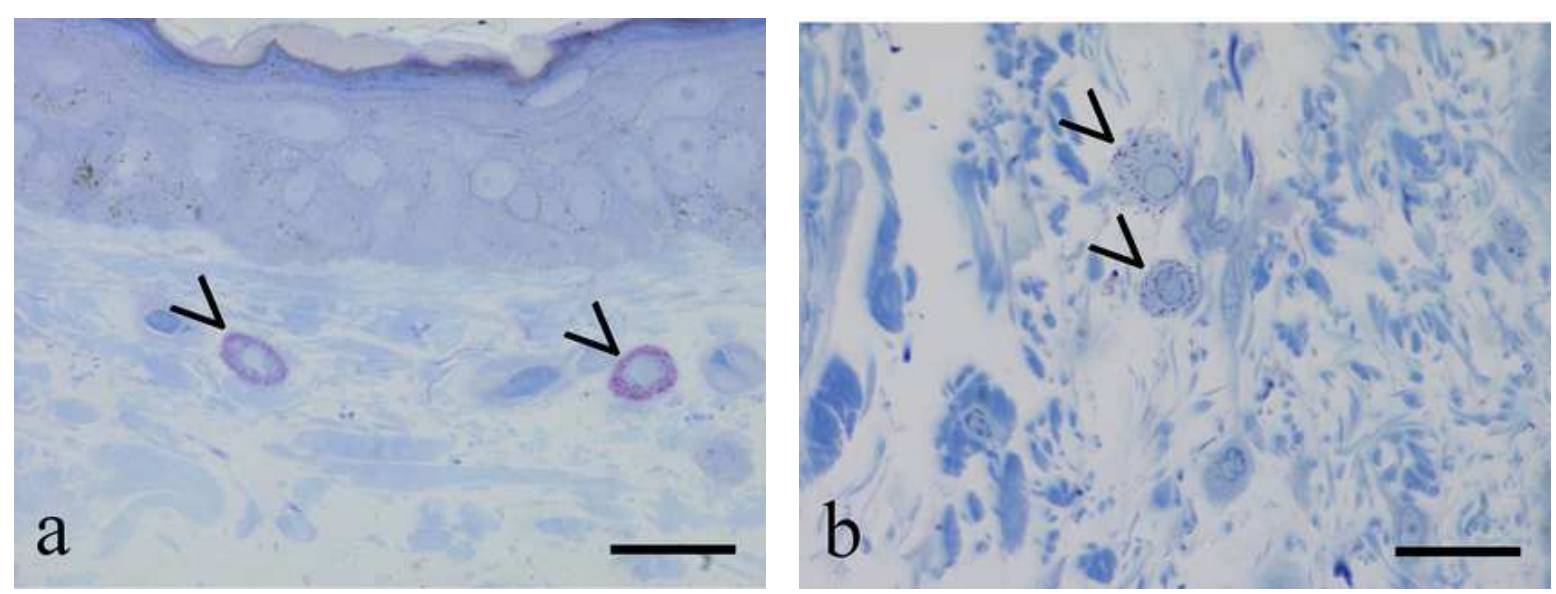

Fig. 5. Photomicrographs of toluidin blue-stained cultured skin biopsies treated with compound 48/80. (a) control; (b) treated (10 mg $\mathrm{mL}^{-1}$ for $20 \mathrm{~min}$ ). Mast cells are indicated by arrowheads. Scale $=20 \mu \mathrm{m}$

\section{Keratinocyte Proliferation}

Proliferating keratinocytes were observed in the basal layer of epidermis until D4 of culture, by means of Ki67 immunostaining (Fig. 3). D7 samples did not show positively stained cells. Proliferating keratinocytes (number of Ki67-positive cells as a percentage of the total number of basal cells) were estimated as $11.16 \pm 8.04 \%$ at $\mathrm{D} 0,11.66 \pm 4.15$ at $\mathrm{D} 1$ and $5.18 \pm 7.51$ at D4. Bonferroni's Multiple Comparison Test showed the mean at D0 and D1 to be significantly different from D7 but not from D4 $(\mathrm{p}<0.001)$.

\section{Epidermal Differentiation Markers}

Cytokeratin 14 was observed in all epidermal layers from D0 to D7. Cytokeratin 10 stained all suprabasal layers and maintained its typical staining pattern from D0 to D7. The staining pattern for loricrin was maintained throughout the study and specifically labeled the granular layer of epidermis (Fig. 4).

\section{Effect of Compound 48/80}

Toluidine blue-stained semi-thin sections showed few intracellular and extracellular granules (MC) after compound $48 / 80$ treatment $\left(10 \mathrm{mg} \mathrm{mL}^{-1}\right)$ (Fig. 5).

\section{Discussion}

Here we report the possibility to culture canine full thickness skin biopsies for one week, under serum free conditions, together with a description of the main histopathological features and changes in epidermal thickness, presence of epidermal melanin, keratinocyte proliferation and epidermal differentiation markers. Epidermis was generally well-preserved during the study and necrotic epidermal changes were not encountered in any section. At D7 only occasional pyknotic keratinocytes were detected, pyknosis being a sensitive indicator for low level tissue damage (Tammi et al., 1979). These data, along with assessment of a physiological number of stratified keratinocytes indicate the presence of a normal spinous layer. Although epidermal thickness decreased from D4, we observed a statistically significant decrease on keratinocyte proliferation only at D7. Previous studies on a human full-thickness skin organ culture model reported divergent data on keratinocyte proliferation: Kleszczynski and Fischer (2012) recorded Ki67-positive cells up to $72 \mathrm{~h}$ culture time, followed by a considerable increase in cell damage/apoptosis; Lu et al. (2007) showed that up until 5 days of culture more proliferating than apoptotic cells were detectable. While the aforementioned study did not analyze early time points (such as D1), values of Ki67-positive cells reported by $\mathrm{Lu}$ et al. (2007) at D5 (6\% proliferating cells) were comparable to our findings at D4 (5.18\%). On the contrary, Kleszczynski and Fischer (2012) observed the complete absence of Ki67 immunoreactivity as early as $72 \mathrm{~h}$. Various factors might be responsible for these differences, such as different species and species-related difference in epidermal anatomy (human skin is much more stratified than canine).

Apoptosis increases quite early in human organ culture, although tissue architecture, viability and functionality are reasonably maintained for up to 2 weeks (Lu et al., 2007). Our model showed a decrease in keratinocyte proliferation with no proliferating cells at D7. More studies assessing the effects of different media composition are needed to evaluate whether a longer viability of the model might be obtained.

The main histological impairment of our model pertained to hair follicles. Degenerative changes starting from D4 were present, with matrix cells being the most affected. These are the most delicate proliferating 
compartment of the bulb and give rise to the rest of the unit. Lu et al. (2007) reported viability of cycling follicles after 17 days of culture; Tobin et al. (1993) observed normal hair follicle growth up to 12 days, although some follicles had undergone involution and displayed gross morphologies similar to catagen. Based on the available data, we do not think that a dependable or unique hypothesis could be formulated. The presence of residual subcutaneous fat tissue might have impaired the microenviroment of the lower portion of the follicles by preventing diffusion of one or more culture medium components. Improved resection of the subcutaneous fat might resolve the problem; supplementation with serum is to be avoided in order to maintain well-controlled conditions. Serum-free media are commonly used for human cell cultures with good results (Lu et al., 2007, Kleszczynski and Fischer, 2012; de Jesus Ribeiro et al., 2005).

Evaluation of pigmentation evidenced no changes throughout the study. We assessed pigment content within a defined area of epidermis avoiding to select the stratum corneum. Despite the decrease in epidermal thickness during the study, melanin density remained constant; this latter finding may relate to the fact that most of the pigment resides on the basal layer of epidermis and would be the last to be lost during atrophy. Indeed, previous studies both in guinea pigs and humans showed that melanin synthesis proceeds up to 7 days of skin organ culture (Imokawa and Motegi, 1993; de Jesus Ribeiro et al., 2005). Thus our findings substantiate previous reports and indirectly confirm a maintained physiology of the explanted canine skin, with activity of melanocytes reportedly dependent on keratinocyte viability (Duval et al., 2002).

Normal physiology of epidermis relies also on keratinocyte proliferation and differentiation (Xu et al., 2012). Immunostaining of our samples with different epidermal differentiation-associated factors (cytokeratin 10 , cytokeratin 14 and loricrin) revealed all markers maintained the same pattern at D0 through D7. Thus, basal keratinocytes continue to differentiate and form the stratified squamous epithelium.

MC degranulation was observed in semithin toluidine blue-stained skin sections treated with compound 48/80, which also induces MC degranulation ex vivo in the rainbow trout isolated intestine (Manera et al., 2011). Ex vivo MC modulation can also be induced via cannabinoid receptor 1 pharmacological blockade or gene silencing, both of which significantly stimulate MC degranulation within the connective tissue sheath of human hair follicles (Sugawara et al., 2012).

This study represents the first attempt to culture dog skin and documents good preservation of most cutaneous structures until D7 in serum-free conditions. Culture methods are considered successful when morphological and physiological activities are maintained under controlled conditions.

\section{Conclusion}

Further studies are necessary to better understand canine skin organ culture, e.g. refining experimental procedures to establish longer-term cultures, as in human medicine (Bagabir et al., 2012; Lu et al., 2007). Future investigations should aim to delve deeper into canine skin organ culture characterization, to include examining the effect of natural and synthetic molecules on epidermis features as well as MC behavior within the natural microenvironment. Achieving these goals will open new possibilities into the study of skin pathophysiology and identification of new dermal therapeutics.

\section{Acknowledgement}

Authors are indebted to Prof. Ralf Paus for having suggested the general design of the current assay and for supporting its establishment. Moreover, authors gratefully acknowledge Stephen D. Skaper (Department of Pharmaceutical and Pharmacological Sciences, University of Padua) for his valuable help in language assistance and proof reading of the manuscript.

\section{Author Contributions}

Francesca Abramo, Silvia Vidali and Vincenzo Miragliotta: Contributed to the conception and design of the study, data analysis and writing of the manuscript and final critical revision of the manuscript.

Andrea Pirone and Carla Lenzi: Involved in histological data production and acquisition.

Maria Federica della Valle: Final critical revision of the manuscript.

Iacopo Vannozzi: Selected the animals and provided samples to be included in the study and final critical revision of the manuscript.

\section{Competing Interests}

MFdV is a scientific consultant for CeDIS (Science Information and Documentation Centre), Innovet Italia srl. None of the other authors declare a conflict of interest.

\section{References}

Abramo, F., L. Campora, F. Albanese, M.F. Della Valle and L. Cristino et al., 2014. Increased levels of palmitoylethanolamide and other bioactive lipid mediators and enhanced local mast cell proliferation in canine atopic dermatitis. BMC Vet. Res., 10: 21-21. DOI: 10.1186/1746-6148-10-21 
Bagabir, R., F. Syed, R. Paus and A. Bayat, 2012. Longterm organ culture of keloid disease tissue. Exp. Dermatol., 21: 376-81. DOI: $10.1111 /$ j.1600-0625.2012.01476.x

Beaven, E.P. and A.J. Cox, JR. 1965. Organ Culture of Human Skin. J. Invest. Dermatol., 44: 151-6.

Campora, L., V. Miragliotta, E. Ricci, L. Cristino and V. di Marzo et al., 2012. Cannabinoid receptor type 1 and 2 expression in the skin of healthy dogs and dogs with atopic dermatitis. Am. J. Vet. Res., 73: 988-95. DOI: 10.2460/ajvr.73.7.988

Caron, G.A., 1968. Organ culture of normal and psoriatic skin. Arch. Dermatol., 97: 575-86. DOI: 10.1001/archderm.1968.01610110083014

Cerrato, S., P. Brazis, A. Meana, D. Fondevila and A. Puigdemont, 2012. In vitro development and characterization of canine epidermis on a porcine acellular dermal matrix. Vet. J., 193: 503-7. DOI: $10.1016 /$ j.tvj1.2012.01.031

Dame, M.K., D.M. Spahlinger, M. Dasilva, P. Perone and R. Dunstan et al., 2008. Establishment and characteristics of Gottingen minipig skin in organ culture and monolayer cell culture: Relevance to drug safety testing. In Vitro Cell Dev. Biol. Anim., 44: 245-52. DOI: $10.1007 / \mathrm{s} 11626-008-9091-3$

de Jesus Ribeiro, C., M.T. Ohara and P. Gama, 2005. Alternative model to human skin organ culture: A preliminary study with Leibovitz L15 medium. Microsc. Res. Tech., 66: 139-44. DOI: $10.1002 /$ jemt.20153

Duval, C., N.P. Smit, A.M. Kolb, M. Regnier and S. Pavel et al., 2002. Keratinocytes control the pheo/eumelanin ratio in cultured normal human melanocytes. Pigment Cell Res., 15: 440-6. DOI: 10.1034/j.1600-0749.2002.02055.x

Imokawa, G. and I. Motegi, 1993. Skin organ culture model for examining epidermal melanization. J. Invest. Dermatol., 100: 47-54.

Jassies-Van Der Lee, A., V.P. Rutten, J. Bruijn, T. Willemse and F. Broere, 2014. $\mathrm{CD}^{+}$and $\mathrm{CD} 8^{+}$ skin-associated $\mathrm{T}$ lymphocytes in canine atopic dermatitis produce interleukin-13, interleukin-22 and interferon- $\gamma$ and contain a $\mathrm{CD} 25^{+} \mathrm{FoxP}^{+}$subset. Vet. Dermatol., 25: 456-e72.

DOI: $10.1111 /$ vde. 12140

Kimura, T., M. Sekido, N. Chimura, S. Shibata and N. Kondo et al., 2012. Production of GM-CSF mediated by cysteine protease of Der $f$ in canine keratinocytes. J. Vet. Med. Sci., 74: 1033-6.

DOI: 10.1292/jvms.11-0522

Kleszczynski, K. and T.W. Fischer, 2012. Development of a short-term human full-thickness skin organ culture model in vitro under serum-free conditions. Arch Dermatol. Res., 304: 579-87. DOI: $10.1007 / \mathrm{s} 00403-012-1239-\mathrm{z}$
Lee, J. and K.T. Lim 2010. Inhibitory effect of phytoglycoprotein $(24 \mathrm{kDa})$ on allergy-related factors in compound 48/80-induced mast cells in vivo and in vitro. Int. Immunopharmacol., 10: 591-599. DOI: 10.1016/j.intimp.2010.02.009

Lu, Z., S. Hasse, E. Bodo, C. Rose and W. Funk et al., 2007. Towards the development of a simplified long-term organ culture method for human scalp skin and its appendages under serum-free conditions. Exp. Dermatol., 16: 37-44. DOI: $10.1111 / \mathrm{j} .1600-0625.2006 .00510 . x$

Manera, M., A. Giammarino, C. Borreca, L. Giari and B.S. Dezfuli, 2011. Degranulation of mast cells due to compound $48 / 80$ induces concentrationdependent intestinal contraction in rainbow trout (Oncorhynchus mykiss Walbaum) ex vivo. J. Exp. Zool. A, 315: 447-57. PMID: /21678562

Marsella, R., T. Olivry, D.N. Carlotti and ITFCAD, 2011. Current evidence of skin barrier dysfunction in human and canine atopic dermatitis. Vet. Dermatol., 22: 239-48. DOI: 10.1111/j.1365-3164.2011.00967.x

Marsella, R., C.A. Sousa, A.J. Gonzales and V.A. Fadok, 2012. Current understanding of the pathophysiologic mechanisms of canine atopic dermatitis. J. Am. Vet. Med. Assoc., 241: 194-207.

DOI: 10.2460/javma.241.2.194

Prose, P.H., A.E. Friedman-Kien and S. Neistein, 1967. Ultrastructural studies of organ cultures of adult human skin. In vitro growth and keratinization of epidermal cells. Lab. Invest., 17: 693-716.

Sarkany, I., K. Grice and G.A. Caron, 1965. Oroan culture of adult human skin. Br. J. Dermatol., 77: 65-76. DOI: 10.1111/j.1365-2133.1965.tb14602.x

Serra, M., P. Brazis, A. Puigdemont, D. Fondevila and V. Romano et al., 2007. Development and characterization of a canine skin equivalent. Exp. Dermatol., 16: 135-42. DOI: $10.1111 /$ j.1600-0625.2006.00525.x

Shibata, S., S. Maeda, S. Maeda, N. Chimura and N. Kondo et al., 2010. Augmentation of CCL17 and CCL2 8 gene expression by TNF- $\alpha$, IL- $1 \beta$, or IFN- $\gamma$ in cultured canine keratinocytes. Res. Vet. Sci., 88: 422-6. DOI: 10.1016/j.rvsc.2009.11.011

Sugawara, K., T. Biro, D. Tsuruta, B.I. Toth and A. Kromminga et al., 2012. Endocannabinoids limit excessive mast cell maturation and activation in human skin. J. Allergy Clin. Immunol., 129: 726-738.

Tammi, R., C.T. Jansen and R. Santti, 1979. Histometric analysis of human skin in organ culture. J. Invest. Dermatol., 73: 138-40. DOI: $10.1111 / 1523-1747 . e p 12581579$ 
Tobin, D.J., N. Mandir and R. Dover, 1993. Morphological analysis of in vitro human hair growth. Arch Dermatol. Res., 285: 158-64. DOI: $10.1007 / \mathrm{BF} 01112919$

Wang, Y.H., Y. Tache, A.G. Harris, W. Kreutner and A.F. Daly et al., 2005. Desloratadine prevents compound 48/80-induced mast cell degranulation: Visualization using a vital fluorescent dye technique. Allergy, 60: 117-24. DOI: $10.1111 / \mathrm{j} .1398-9995.2004 .00641 . x$

Wilkinson, J.E., C. Smith, M. Suter and R.M. Lewis, 1987. Long-term cultivation of canine keratinocytes. J. Invest. Dermatol., 88: 202-6.
Xu, W., S. Jong Hong, S. Jia, Y. Zhao and R.D. Galiano et al., 2012. Application of a partial-thickness human ex vivo skin culture model in cutaneous wound healing study. Lab. Invest., 92: 584-99. DOI: $10.1038 /$ labinvest.2011.184

Yagihara, H., T. Okumura, E. Shiomi, N. Shinozaki and S. Kuroki et al., 2011. Reconstruction of stratum corneum in organotypically cultured canine keratinocyte-derived CPEK cells. Vet. Res. Commun., 35: 433-7.

DOI: $10.1007 / \mathrm{s} 11259-011-9477-7$ 